\title{
Characterization of ambient seismic noise near a deep geothermal reservoir and implications for interferometric methods: a case study in northern Alsace, France
}

Maximilien Lehujeur*, Jérôme Vergne, Jean Schmittbuhl and Alessia Maggi

* Correspondence: lehujeur@unistra.fr IPGS, Université de Strasbourg/ EOST, CNRS, 5 rue René Descartes, 67084 Strasbourg Cedex, France

\begin{abstract}
Background: Ambient noise correlation techniques are of growing interest for imaging and monitoring deep geothermal reservoirs. They are simple to implement and can be performed continuously to follow the evolution of the reservoir at low cost. However, these methods rely on assumptions of spatial and temporal uniformity of seismic noise sources. Violating them can result in misinterpretation of seismic velocities owing to preferential noise propagation directions.

Methods: Using several years of seismic data recorded around the two geothermal sites of Soultz-sous-forêts and Rittershoffen in northern Alsace, France, we propose a detailed characterization of the spatial and temporal properties of the high frequency seismic noise $(0.2$ to $5 \mathrm{~Hz})$. We consider two fundamental properties of the cross correlation functions (CCFs) of ambient noise. Firstly, the reliability of the Green's function reconstruction, an important indicator for tomographic studies. Secondly, the temporal repeatability of the CCFs between 0.2 and 0.5 seconds.

Results and conclusions: At periods below 1s, we observe a sharp decrease in signal to noise ratio resulting from the non uniform distribution of anthropogenic sources. At periods above 1s, we show that the high directivity of the northern Atlantic microseismic peak biases the CCFs' phase significantly. We show that nocturnal noise is the most suited for temporal analysis of the CCFs. Using nocturnal noise, we should be able to monitor temporal variations induced by the geothermal activities inside the reservoir.
\end{abstract}

Keywords: Ambient noise; Cross-correlation; Surface waves; Anthropogenic noise

\section{Springer}

\section{Background}

Projects dedicated to the exploitation of deep geothermal resources need to probe the upper crustal structure of the targeted area in order to characterize the reservoir and its relation to pre-existing geological formations. Active seismic sounding is a commonly used approach; its dense spatial and temporal sampling provides high-resolution images of the reflectivity of the subsurface layers and of fault geometry. However, such seismic data are not readily available everywhere, and acquisition of new data, especially in 3D, is often very expensive compared to the profitability of geothermal resources. Its high cost 
also excludes using repeated active seismic sounding to follow the evolution of the medium during production.

Images of the upper crustal structure can also be obtained from tomographic inversion of arrival times of natural or induced local earthquakes. These inversions can be repeated over time to map velocity changes (e.g., Calò et al. 2011; Calò and Dorbath 2013) much more cheaply than active seismic sounding. Producing good-quality tomographic images of geothermal reservoirs using arrival times requires having induced seismicity around the wells, but geothermal operators need to minimize induced seismicity to reduce the seismic risk associated with their exploitation. The need to minimize seismic risk excludes using time-lapse arrival-time tomography for continuous reservoir imaging.

Over the past 10 years, another promising passive seismic imaging technique has emerged. Known as 'ambient noise tomography', it uses seismic noise as a permanent source of energy that propagates through the target region. The cross-correlation function (CCF) of long records of seismic noise at a pair of stations provides an estimation of Green's function between them (Lobkis and Weaver 2001; Shapiro and Campillo 2004; Sabra et al. 2005a). The resulting correlogram is similar to the signal that would be obtained if an impulsive source occurred at one station and was recorded by the other one. This method allows us to perform tomographic studies using all possible pairs of stations over a network (Shapiro et al. 2005; Sabra et al. 2005b). The resolution of the recovered seismic velocity models only depends on the geometry of the stations. This technique has been widely applied at various scales, from the structure of the mantle using worldwide broadband stations (Poli et al. 2012; Lin and Tsai 2013; Lin et al. 2013) to laboratory samples using piezoelectric sensors (e.g., Lobkis and Weaver 2001; Derode et al. 2003a, b; Larose et al. 2007). At the local scale, the method has been applied in various environments from offshore oil reservoirs (Bussat and Kugler 2011; Mordret et al. 2013) to active volcanic systems (Brenguier et al. 2008, 2011). Although the applicability of ambient noise tomography in the context of geothermal reservoirs is still under debate, at least one application has already been attempted using a local network of short-period seismometers around the geothermal site of Soultz-sousForêts (Calò et al. 2013). Beyond its use in seismic tomography, the continuous nature of seismic noise can also be exploited to observe subtle variations in the seismic velocity or the diffracting character of the crust. For example, Brenguier et al. $(2008,2011)$ and Obermann et al. (2013) were able to produce 4D pictures of the Piton de la Fournaise volcano by applying interferometric analysis to the coda part of the correlograms.

The seismic noise-based methods described above all rely on strong assumptions concerning the noise sources. For tomography applications, noise sources should be homogeneously distributed (Lobkis and Weaver 2001; Roux et al. 2005). Under this assumption, only the sources located in a narrow area along the continuation of the path joining the two stations contribute to the recovered Green's function (Roux et al. 2004; Sabra et al. 2005c; Larose 2005; Gouédard et al. 2008). For applications that monitor time-dependent perturbations of the medium, noise sources may be inhomogeneously distributed, but in this case, they must be repeatable. If the seismic noise sources move too much over time, the resulting changes in the signal could be mistaken for perturbations of the medium (Hadziioannou et al. 2009; Weaver et al. 2011).

The consequences of violating these assumptions have been studied theoretically and numerically using synthetic data (Weaver et al. 2009; Froment et al. 2010). Although 
the seismic noise distribution on Earth is rarely homogeneous in time and space, the CCFs approximate Green's functions correctly if the inter-station distances are long and the azimuthal distribution of the noise is smooth. However, when the noise source distribution is highly heterogeneous, some studies using real data report significant bias and incorrect estimation of seismic velocities between the station pairs (Pedersen and Krüger 2007).

Here, we focus on the application of the ambient noise correlation technique in the context of a geothermal reservoir (i.e., a kilometer scale) using seismic data around geothermal sites in northern Alsace. We analyze the characteristics of the seismic noise in the period range between 0.2 and $5 \mathrm{~s}(0.2$ to $5 \mathrm{~Hz})$. We then study the correlograms of ambient noise records between pairs of stations and show how the seismic noise distribution influences the quality and reliability of the reconstructed Green's functions in this particular period range. Finally, we examine the stability of the high-frequency CCF coda for future analysis of temporal changes within the reservoir.

\section{Data}

The Upper Rhine Graben concentrates several sites dedicated to the exploration and exploitation of deep geothermal energy. Northern Alsace hosts both the prototype site of Soultz-sous-Forêts which was initiated about 20 years ago and a recent industrial project in Rittershoffen (ECOGI), $10 \mathrm{~km}$ to the southeast, which started in 2012 and is expected to reach its exploitation phase in 2015. Seismometers have been deployed around both sites to monitor the natural and induced seismicity (Figure 1). They form a permanent network of 12 short-period stations equipped with 1-Hz L4C sensors and digitizers sampling at rates from 100 to $200 \mathrm{~Hz}$. In this study, we use the high-quality continuous recordings available since 2010 for the Soultz-sous-Forêts network and since 2012 for the Rittershoffen network. We also include data from a temporary network of 16 short-period sensors (1-Hz corner frequency) installed in May 2013 by the Karlsruhe Institute of Technology and Ecole et Observatoire des Sciences de la Terre of Strasbourg to densify the permanent network around the site of Rittershoffen (purple network, Figure 1; Gaucher et al. 2013) during stimulation of the GRT1 well. Taken altogether, these stations form a $15-\mathrm{km}$-wide seismic array, whose station spacing ranges from 1 to $15 \mathrm{~km}$.

In order to extend our understanding of the origins of seismic noise to higher frequencies, we deployed two small aperture arrays, ARIT and AKUL, close to the location of the permanent stations RITT and KULH (Figure 1). The arrays operated for 2 months during fall 2012. Each array contained six vertical short-period sensors (1-Hz corner frequency) with one three-component $\mathrm{L} 4 \mathrm{C}$ sensor at the center. All the sensors were connected by cables to a central acquisition system that provided a common time reference for the nine recorded channels. They were deployed in a helical configuration with a 300-m maximum aperture.

\section{Methods}

\section{Frequency content and temporal variability of seismic noise}

Empirical Green's functions constructed from the correlation of vertical component ambient seismic noise records are dominated by surface waves, because most noise 


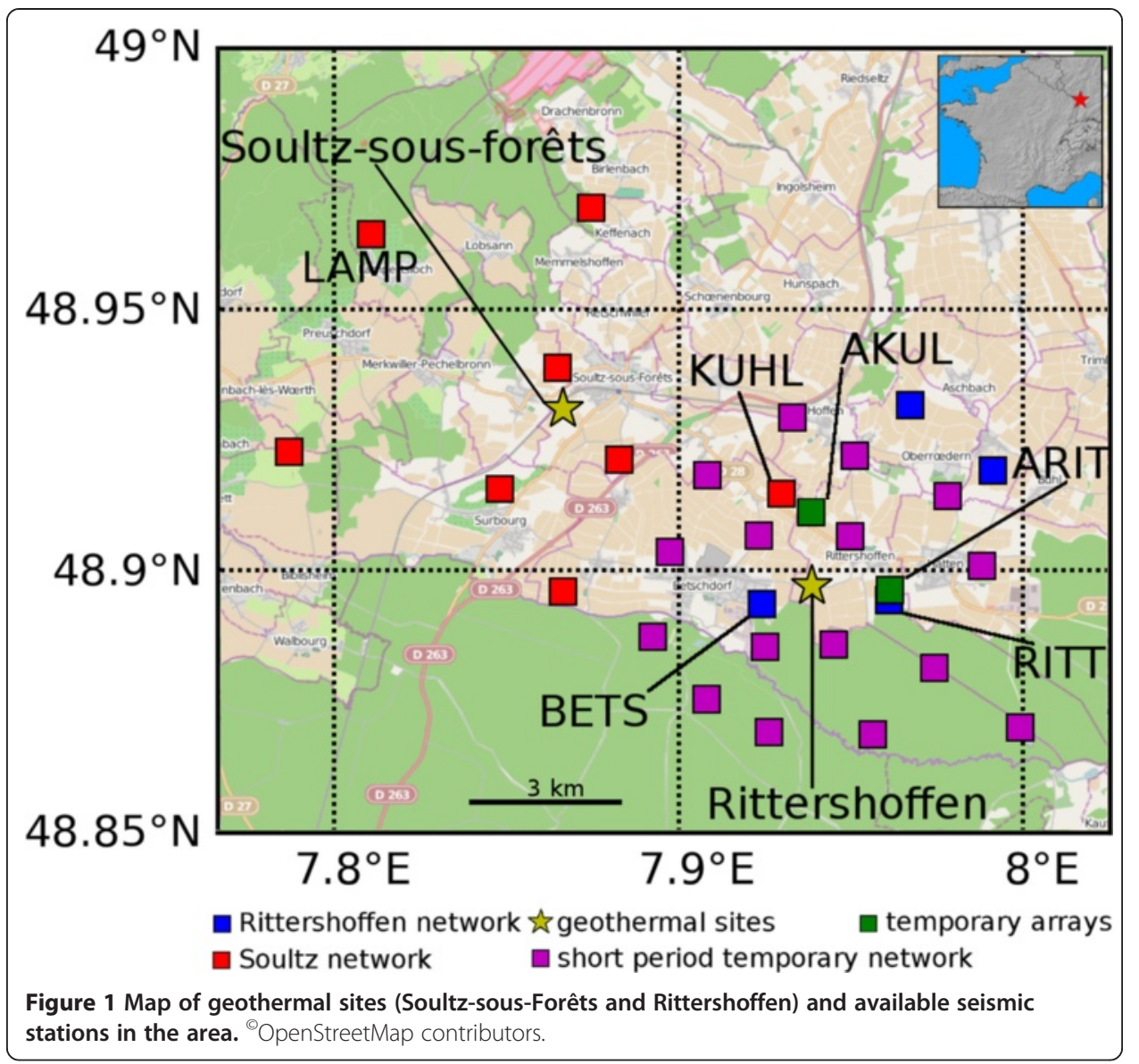

sources occur close to the Earth's surface. Therefore, the dispersive character of the Rayleigh waves is the primary information that can be extracted from correlograms (Campillo et al. 2011). Dispersion measurements for each pair of stations (group or phase dispersion curves) can be regionalized to provide spatial variations of surfacewave velocities at each period. Inversion can then be used to convert surface-wave velocities as a function of period to $\mathrm{S}$-wave velocities as a function of depth (the longer the surface-wave period, the greater the investigation depth). At Soultz-sous-Forêts and Rittershoffen, the reservoir lies between 2- and 5-km depths. In order to map S-wave velocities from the surface to that depth using the dispersive character of the Rayleigh waves, we must work in the period range of 0.2 to $5 \mathrm{~s}$. This range is compatible with the bandwidth of our seismometers (cutoff period of $1 \mathrm{~s}$ ) and benefits from low instrumental noise.

Between 0.2 and $5 \mathrm{~s}$, seismic noise has differing origins and properties. For periods above $2 \mathrm{~s}$, seismic noise spectra everywhere on Earth contain a broad, highly energetic peak called the 'secondary micro-seismic peak.' This peak results from pressure variations on the sea bottom induced by interferences of oceanic waves traveling in opposite directions (Longuet-Higgins 1950). A few dominant zones in the north Atlantic (south of Greenland, along the Canadian coasts and around the mid-Atlantic ridge) generate most of the secondary micro-seismic peak energy recorded in Europe (Gutenberg 1936; Kedar et al. 2008; Sergeant et al. 2013). 
At periods below $2 \mathrm{~s}$, numerous phenomena are responsible for the observed seismic noise. They can be split into two categories: natural sources, among which the wind acting on trees or structures sealed into the ground in the vicinity of the recording stations (Withers et al. 1996; Bonnefoy-Claudet et al. 2006), and anthropogenic sources like road traffic, industries, or other types of human activities (McNamara and Buland 2004; Groos and Ritter 2009). As seismic noise from these high-frequency sources propagates only to local distances, its characteristics change from one region to another. A region-specific analysis of the high-frequency noise spectrum is therefore recommended before applying ambient noise correlation techniques (Campillo et al. 2011).

Figure 2 presents spectrograms of the noise recorded at station RITT. The columns in each spectrogram represent the amplitude of the Fourier transform of the ground acceleration computed for $1 \mathrm{~h}$ of signal. The 1 - to 10-s period range (above the white dotted line) is dominated by the secondary micro-seismic peak; its maximum amplitude occurs between 2 and $7 \mathrm{~s}$. The amplitude of this noise is independent of the recording site (see Additional file 1 and Additional file 2: Figure S1), proving that it is produced by distant sources and is recorded coherently over the whole network. We observe an annual periodicity, with an increase of energy during the winter (white arrows labeled $\mathrm{W})$. Below $1 \mathrm{~s}$, the noise is very different and presents a strong daily periodicity with a decrease of energy during the night (white arrows labeled $\mathrm{N}$ ) and at noon (white arrows labeled 12). We also observe a weekly periodicity with significant noise reduction

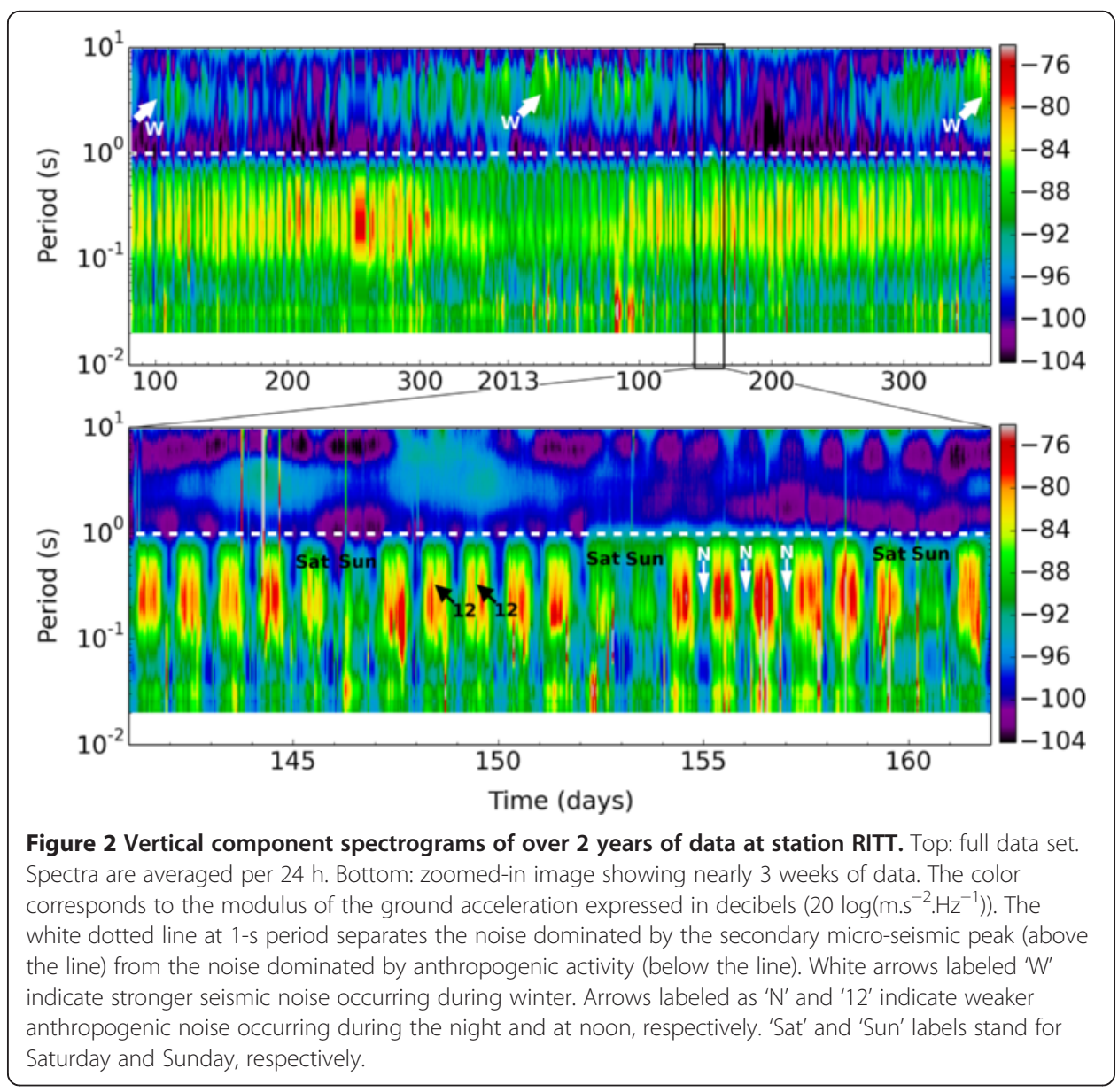


during the weekend (labels 'Sat' and 'Sun'). These periodicities underline the dominant role played by anthropogenic sources in this period band. Even though the same overall features are observed on most stations, the energy and detailed characteristics are highly dependent on the recording site, confirming that this high-frequency noise is generated close to the stations (see Additional file 1 and Additional file 2).

\section{Spatial distribution of seismic noise}

To estimate the spatial origin of the seismic noise over the whole period range of interest, we apply a classical beamforming technique (e.g., Rost and Thomas 2002) to the local monitoring network and the small aperture arrays. This technique allows us to determine the dominant back azimuth and phase velocity of an incoming seismic wave, so long as the network's station spacing is less than half the wavelength. By applying the method on a sliding window, we can estimate the directivity and phase velocity of the noise as a function of time. The longer the period of the noise to be processed, the wider the array must be.

The permanent network of Soultz-sous-Forêts can be used as a single array for periods between 2 and $5 \mathrm{~s}$. We estimate the most probable incoming direction and phase speed of the noise over 4 years of continuous records divided into 15-min windows. The results are given as a probability density function in the phase speed-back azimuth domain. Figure 3 shows an example for 2-s period. At this period, we observe a dominant phase velocity of approximately $3 \mathrm{~km} / \mathrm{s}$, corresponding to the average phase speed of Rayleigh waves under the Soultz network. Over $95 \%$ of the noise arrives from back azimuths between $265^{\circ}$ and $345^{\circ}$, which correspond to the direction of the northern Atlantic Ocean. The other periods between 2 and $5 \mathrm{~s}$ yield similar results. If we group the beamforming results as a function of the month of the year, we observe a coherent annual variation of $\pm 5^{\circ}$, which is probably related to small seasonal variations in the location of the noise sources in the Atlantic Ocean (Figure 3, bottom).

Below $1 \mathrm{~s}$, beamforming can no longer be performed on the full network because the station spacing is too large compared to the wavelength of the seismic noise, which results in aliasing effects. For this reason, we use the two small aperture arrays AKUL and ARIT to identify the origin of the noise at periods below $1 \mathrm{~s}$. We estimate the most probable incoming direction and phase speed of the noise from 2 months of continuous recording divided up into 15-s windows. Figure 4 presents the results of the beamforming analysis at a period of $0.3 \mathrm{~s}$. The radial histograms of measured back azimuths are normalized to the total number of 15-s windows and superimposed on the map of the area at the array locations (left side of Figure 4). Note that these histograms do not provide any information about the energy of the seismic sources but only their statistical distribution in azimuth. The azimuthal distribution of seismic sources is heterogeneous with few narrow peaks, indicating that the high-frequency noise around the arrays is mostly generated in two zones that roughly correspond to the neighboring villages. Furthermore, the relative weight of these zones varies with the time of day (right side of Figure 4). For instance, the source detected by array AKUL at back azimuth $140^{\circ}$ - the village of Rittershoffen - becomes prominent during the day. Other sources can only be observed at night, such as the one detected by array AKUL at back azimuth $50^{\circ}$, which does not point towards any particular village. These sources may be continuous in nature but are masked during the day by other dominant sources. 

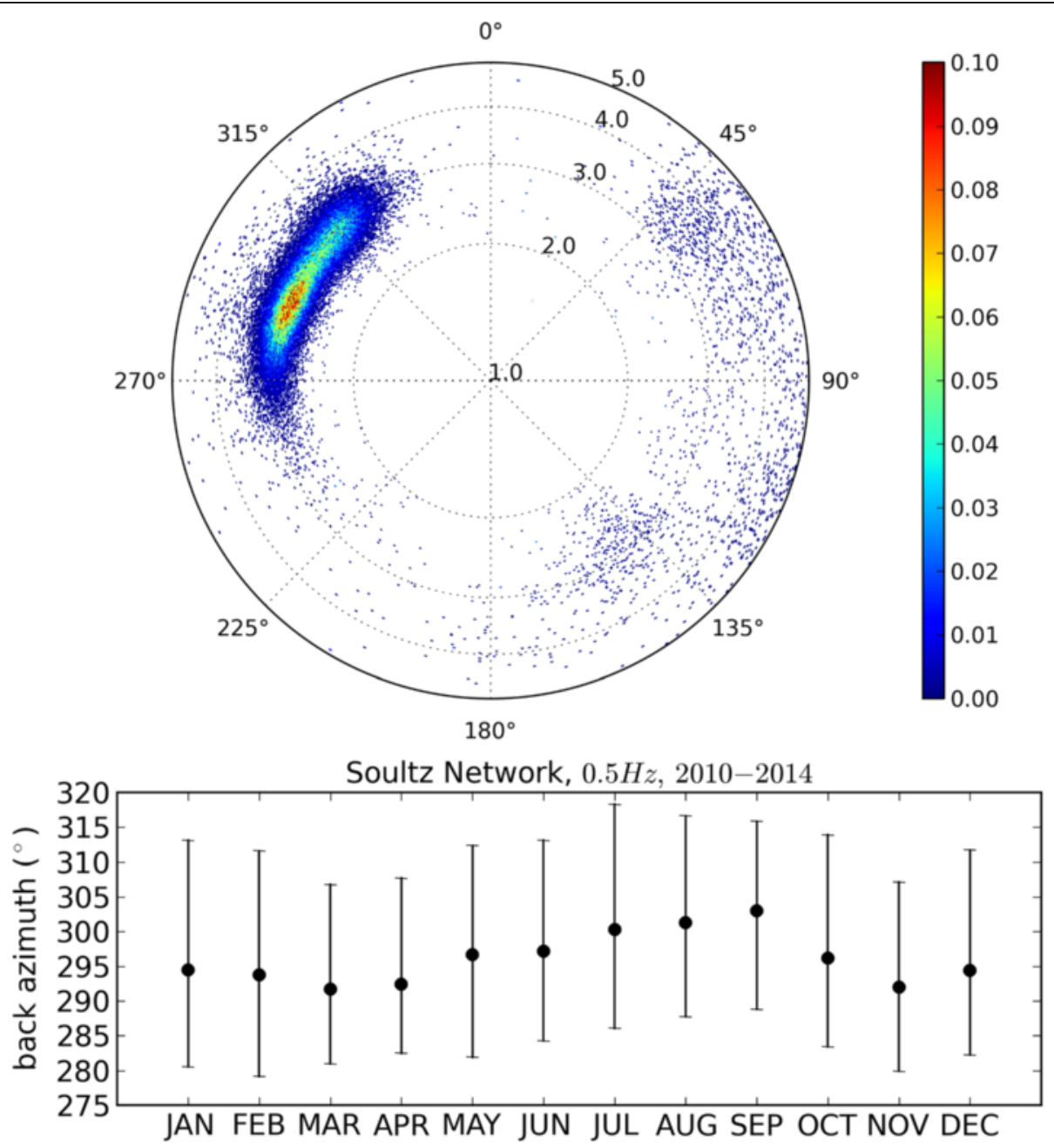

Figure 3 Beamforming analysis performed on the Soultz-sous-Forêts network. About 4 years of data have been divided up into 15-min windows and filtered around 1s. Top: probability density function (color code) of the estimated phase speed (expressed in $\mathrm{km} / \mathrm{s}$ and displayed as the radius) and back azimuth (measured clockwise from the north in degrees) of the noise. Bottom: detail of the variation of the dominant back azimuth measured using the 4 years of data grouped by month (all January months together, etc.). Error bars delimitate the $16 \%$ and $84 \%$ percentiles of the statistical distribution of detected back azimuths.

\section{Results and discussion}

\section{Cross-correlation functions and dispersion measurements}

We compute CCFs for each possible pair of stations of the full network (excluding the small aperture arrays). Each 1-h-long segment of noise is processed individually prior to correlation (see Additional file 1 and Additional file 3). Following the sign convention proposed by Stehly et al. (2006), we systematically correlate the noise recorded at the eastern station of a pair with the western one. Consequently, the noise sources occurring west (resp. east) to the two stations affect the positive (resp. negative) part of the CCF. We band-pass filter the CCFs in three period ranges and represent them according to the inter-station spacing (hodograms; Figure 5). Between 1.25 and $5 \mathrm{~s}(0.2$ to $0.8 \mathrm{~Hz}$; Figure 5, top left), the Rayleigh waves can be unambiguously identified on almost all station pairs. The stronger amplitudes of the positive parts of the correlations 


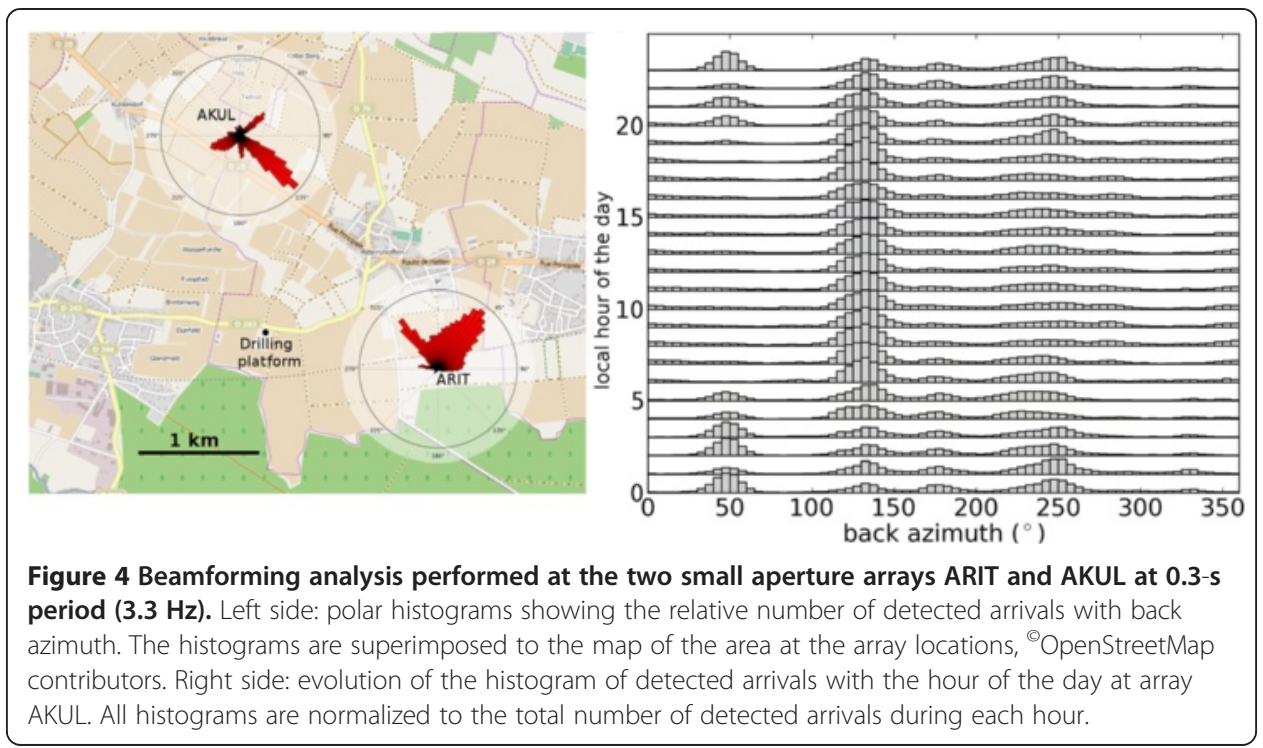

are a consequence of the directivity of the seismic noise (Stehly et al. 2006). Indeed, as shown by the beamforming analysis, this noise mainly originates from the northern Atlantic Ocean located west-northwest of the network. Around $1 \mathrm{~s}$ (Figure 5, top right), the Rayleigh waves can still be followed on the hodogram. The increased symmetry indicates that, in this period range, noise sources are more uniformly distributed around the network or that the noise energy is efficiently distributed through the medium via scattering. However, the signal-to-noise ratio is significantly lower than that in the 1.25- to 5-s period range. Finally, at periods below $1 \mathrm{~s}$ (Figure 5, bottom left), no coherent wave field can be clearly observed, and the signal-to-noise ratio is very low.

These observations are confirmed by the dispersion analysis performed on individual CCFs. We measure the Rayleigh wave dispersion on the CCFs by frequency time analysis (FTAN), which provides an estimation of the group velocity at each period (e.g., Dziewonski et al. 1969; Bensen et al. 2007). Figure 5 (bottom right) illustrates a typical dispersion diagram obtained for the station pair KUHL-BETS. Despite its noisy aspect, we can clearly identify the dispersion of the fundamental mode Rayleigh wave at periods longer than $1 \mathrm{~s}$ (solid black curve on Figure 5, bottom right). This mode can be easily identified on most station pairs. The dispersion diagrams change markedly around $1 \mathrm{~s}$, and we cannot estimate group velocities at shorter periods. This inability occurs regardless of the chosen station pair and the time range (from weeks to years) used to compute the CCF. This transition period of approximately $1 \mathrm{~s}$ is similar to the one observed on the seismic noise spectrograms (Figure 2) and corresponds to the transition between the noise originating from oceanic sources and that generated by local anthropogenic activities.

We propose two possible explanations for the poor quality of the reconstructed Green's function at periods below $1 \mathrm{~s}$ : (1) because of attenuation, the noise generated by low-energy local sources cannot travel far enough to be coherently recorded by two separate stations and/or (2) the non-uniform distribution of the local sources limits the reconstruction of Green's function, as theoretically predicted. The consequences of non-uniform noise sources are described in the following section. 


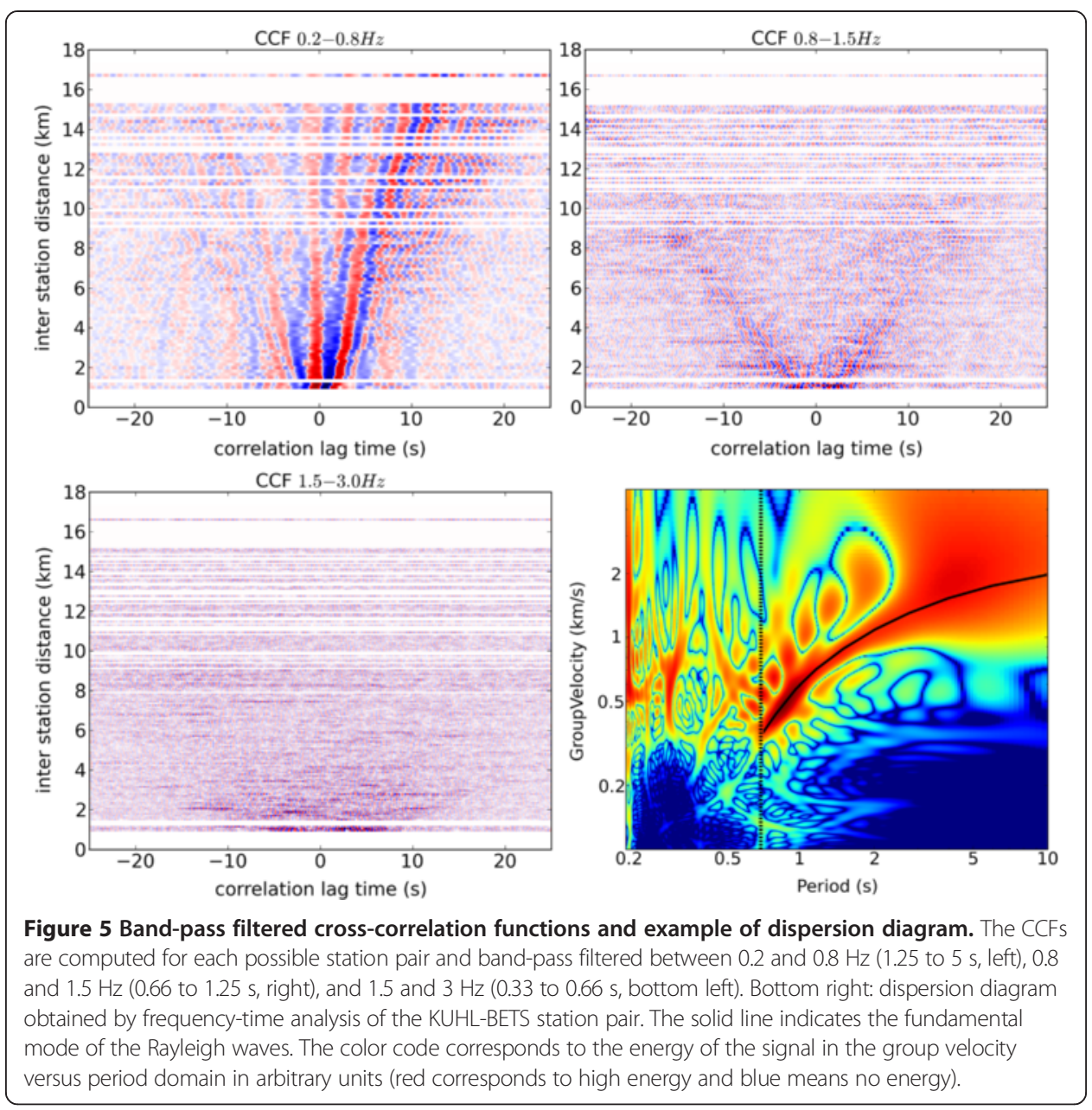

Impact of a directive noise

We observe high signal-to-noise ratio on the phase of the CCFs between 0.2 and $1 \mathrm{~s}$ (Figure 5, top left). We should therefore be able to estimate phase velocities for each station pair and invert them to map their geographical variations. We start by estimating the average Rayleigh wave phase speeds over the whole network at each period by looking for phase alignments among band-pass-filtered CCFs in the time-distance domain (slant stack technique). Figure 6 (top left) presents the CCFs band-pass filtered around $2 \mathrm{~s}$; the dashed lines correspond to the estimated average phase speed $(2.45 \mathrm{~km} / \mathrm{s})$. We then measure the time shift between the phase arrival time picked on each CCF and the one predicted by the average dispersion law (time delay measured between the phase of each CCF and the dashed lines on Figure 6, top left). Negative time shifts correspond to phases arriving sooner than predicted by the reference phase velocity. In the ideal case, these time delays should only be caused by spatial variations of the phase velocities.

We display the measured time shifts as a function of the orientation of the station pair (Figure 6, top right). Because of the chosen orientation convention, phase shifts measured on the positive (resp. negative) part of the CCFs are attributed to azimuths ranging from $180^{\circ}$ to $360^{\circ}$ (resp. $0^{\circ}$ to $180^{\circ}$ ) and are caused by eastward (resp. westward) propagating noise. We observe a sinusoidal shape with a minimum occurring for 


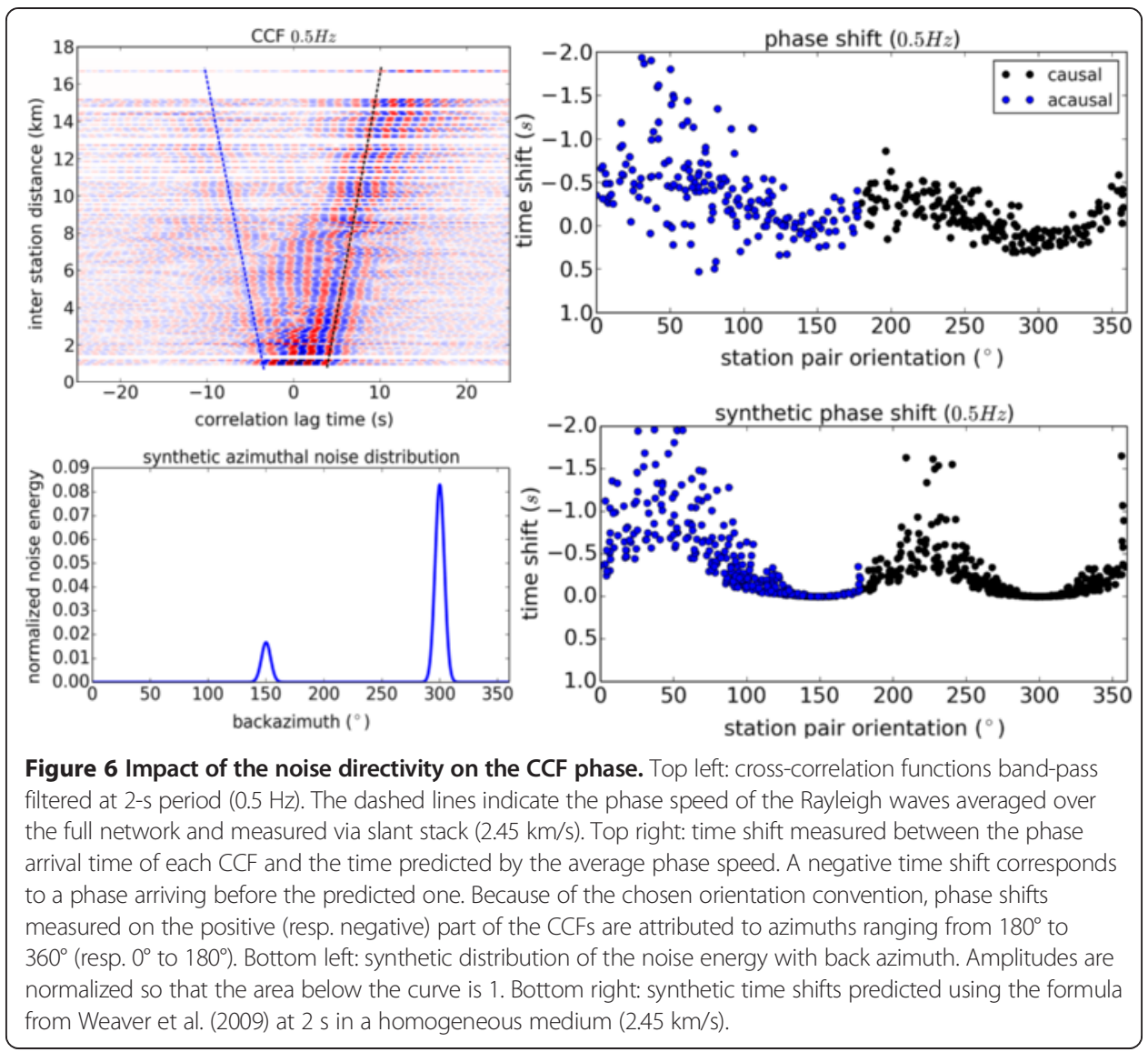

station pairs oriented approximately $300^{\circ}$, corresponding to the case in which the station pairs are aligned with the dominant direction of oceanic noise in this period range (Figure 3). Even though these phase shifts might be due to spatial variations of the phase speed at depth, the sinusoidal-shaped variation combined with a minimum phase shift at $300^{\circ}$ suggests that the phase shifts might in reality be caused by noise directivity. In another context, Pedersen and Krüger (2007) observed apparent variations of the group speed that were actually caused by strong noise directivity.

To corroborate the claim that our phase shifts are essentially caused by noise directivity, we propose a synthetic test with a homogeneous medium (constant phase speed of $2.45 \mathrm{~km} / \mathrm{s}$ at 2-s period). Using the work of Weaver et al. (2009) and Froment et al. (2010), we estimate the theoretical phase shifts predicted by a given azimuthal distribution of the noise energy (ADNE). We find that a simple synthetic ADNE made of two Gaussian functions centered at $150^{\circ}$ and $300^{\circ}$ (the principal back azimuths of the noise directivity observed in Figure 3) reproduces the main features (sinusoidal shape and amplitudes) of the phase shifts measured on real data. Because the synthetic medium is homogeneous, these phase variations can be attributed unambiguously to the noise directivity. Interestingly, as soon as the ADNE contains an isotropic component, however small (even $0.01 \%$ of the dominant arrival), the phase shifts become negligible. This confirms the main conclusion of Weaver et al. (2009) and implies that a complete lack of coherent isotropic noise strengthens our observed phase variations. 
The predicted time shifts in our synthetic test vary from $0 \%$ to $+40 \%$ of the time needed by the phase to travel between two stations, depending on the distance separating the stations and how the pair is oriented with respect to the incoming noise. As our network is quite dense, each grid cell is observed by several station pairs having different orientations and separations. In a tomographic application, one can naively expect to restore the uniform distribution of the noise with azimuth by canceling out the noise-induced phase shift. To understand how the error on the phase of the CCF spreads over the spatial distribution of phase velocities through tomography, we invert the biased synthetic propagation times (Figure 7). We assume a homogeneous a priori velocity of $2.45 \mathrm{~km} / \mathrm{s}$, and set the smoothing distance to $5 \mathrm{~km}$. As the computed phase shifts are all positive, most grid cells exhibit overestimated phase speeds that can reach $30 \%$ more than the true value (green/blue zones). These artefacts are heightened by the large number of station pairs oriented perpendicularly to the dominant noise arrival $\left(300^{\circ}\right)$. The inverse problem also produces zones of underestimated phase speeds (yellow zones) to balance the overestimations and satisfy most of the observations. Future work will focus on how to take this bias into account for a reliable estimation of velocity variations at depth.

\section{Impact of localized and repetitive deterministic high-frequency sources}

Since the energy of short-period noise (below $1 \mathrm{~s}$ ) presents a strong weekly and daily periodicity resulting from human activity (Figure 2, bottom), we average the shortperiod CCFs $(0.2$ to $0.5 \mathrm{~s})$ separately for each day of the week. Figure 8 illustrates the evolution of the CCFs for the 7 days of the week for the station pair RITT-BETS (2.8 $\mathrm{km}$ apart). We observe that the CCFs are very similar from Monday to Friday, while a clear change in phase and amplitude appears on Saturday and Sunday. The stability of the short-period CCFs during weekdays proves that anthropogenic noise can indeed be recorded coherently over several kilometers. We can therefore conclude that

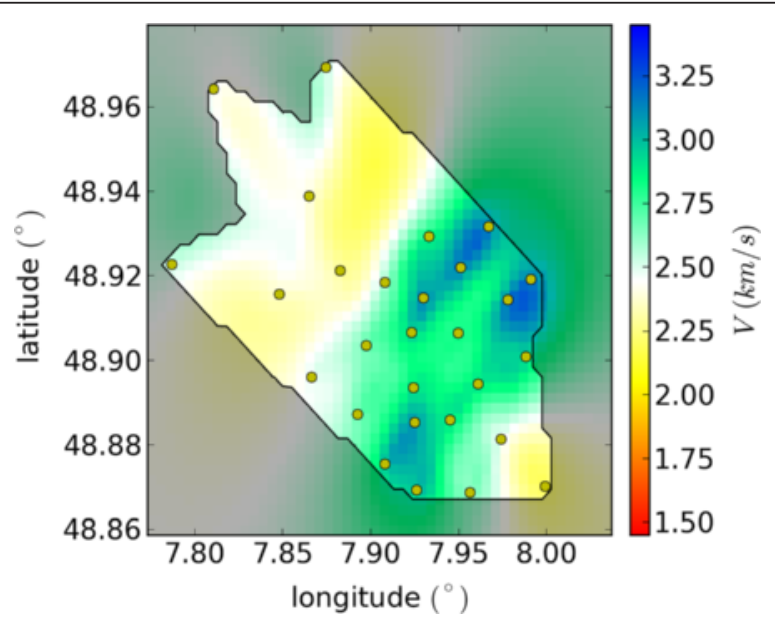

Figure 7 Tomographic inversion of the theoretical time shifts induced by the synthetic anisotropic ADNE. Yellow dots correspond to the stations used in the inversion. The ideal model is homogenous with a phase speed of $2.45 \mathrm{~km} / \mathrm{s}$ (white color). Green/blue (resp. yellow) colors correspond to over-estimated (resp. under-estimated) phase speeds; both causal and acausal time shifts are included in the inverse problem. The black line delimits the resolved zone. 

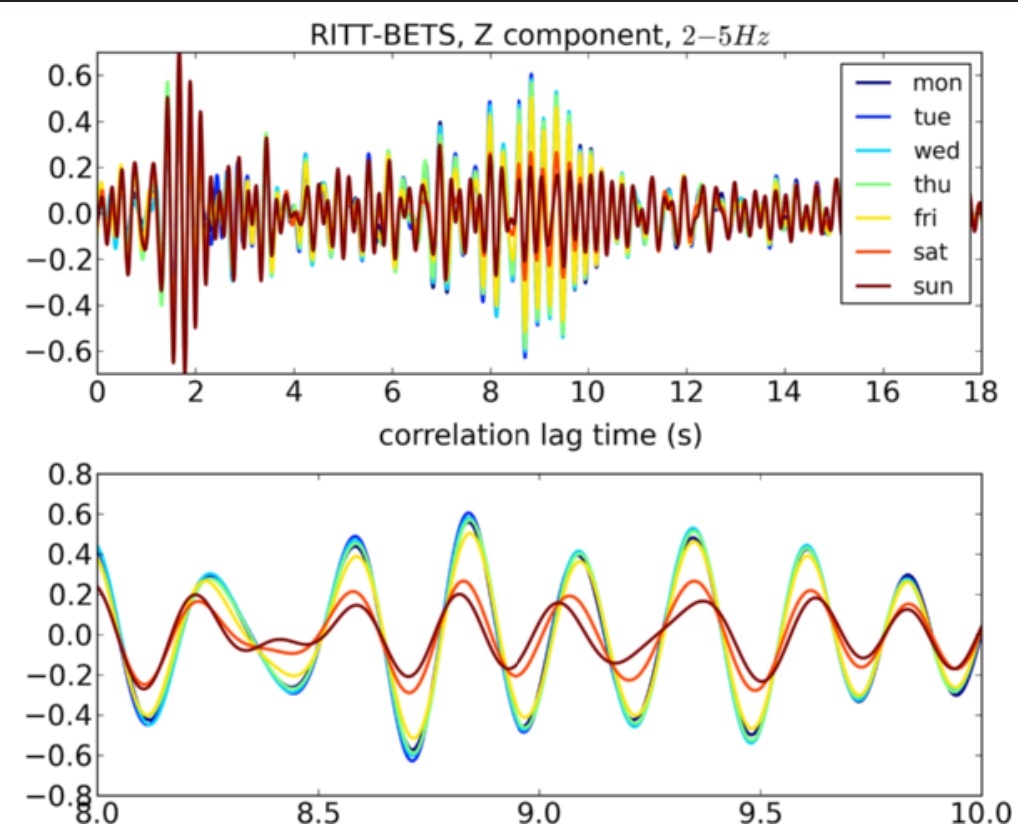

Figure 8 Vertical component cross-correlation functions measured between stations RITT and BETS and band-pass filtered between $\mathbf{0 . 2}$ - and $\mathbf{0 . 5}$-s period. The waveforms are averaged separately depending on the day of the week over 2 years. Bottom: zoom between 8 and $10 \mathrm{~s}$ of correlation lag time.

the reconstruction of the short-period part of Green's function is limited only by the non-uniform distribution of short-period noise sources, and not by attenuation. Furthermore, this observation suggests that the anthropogenic sources are not only localized in space but also repetitive in time.

To refine our understanding of how the variability of anthropogenic sources impacts the correlations, we estimate the variability of the correlation waveform as a function of the local time of the day, restricting the analysis to weekdays (Monday to Friday). To do so, we first isolate the seismic noise recorded from Monday to Friday, then we compute the CCFs in 5-min windows. Finally, we stack the 5-min-long CCFs separately depending on the local time of the day over the whole acquisition period (for instance, we compute all the CCFs of the noise recorded at two stations every working day between local times 08:00 and 08:05 a.m. and stack them). The results for the RITT-BETS case are presented in Figure 9 (left side). Differences can be seen between day and night, with sudden changes of the waveform at 5 a.m. and 9 p.m. (horizontal dashed lines). A very energetic arrival is observed between 5 a.m. and about 7 a.m. (thick black arrow and closeup circle on Figure 9). Its origin has not yet been identified, but this arrival could be caused by a sudden increase of the traffic every morning at the same time along the same roads or by regular start-up times of machines in neighboring industries. The same energetic arrival between 5 a.m. and 7 a.m. is observed for other station pairs but at different correlation lag times, indicating that it is probably caused by a unique localized source. Some arrivals of the early part of the CCF (thick white arrows on Figure 9) can only be seen when the anthropogenic activity decreases (during the nighttime and lunchtime) and could be due to natural (wind related) or continuous anthropogenic noise sources (e.g., pumps or industries in continuous operation). Given 


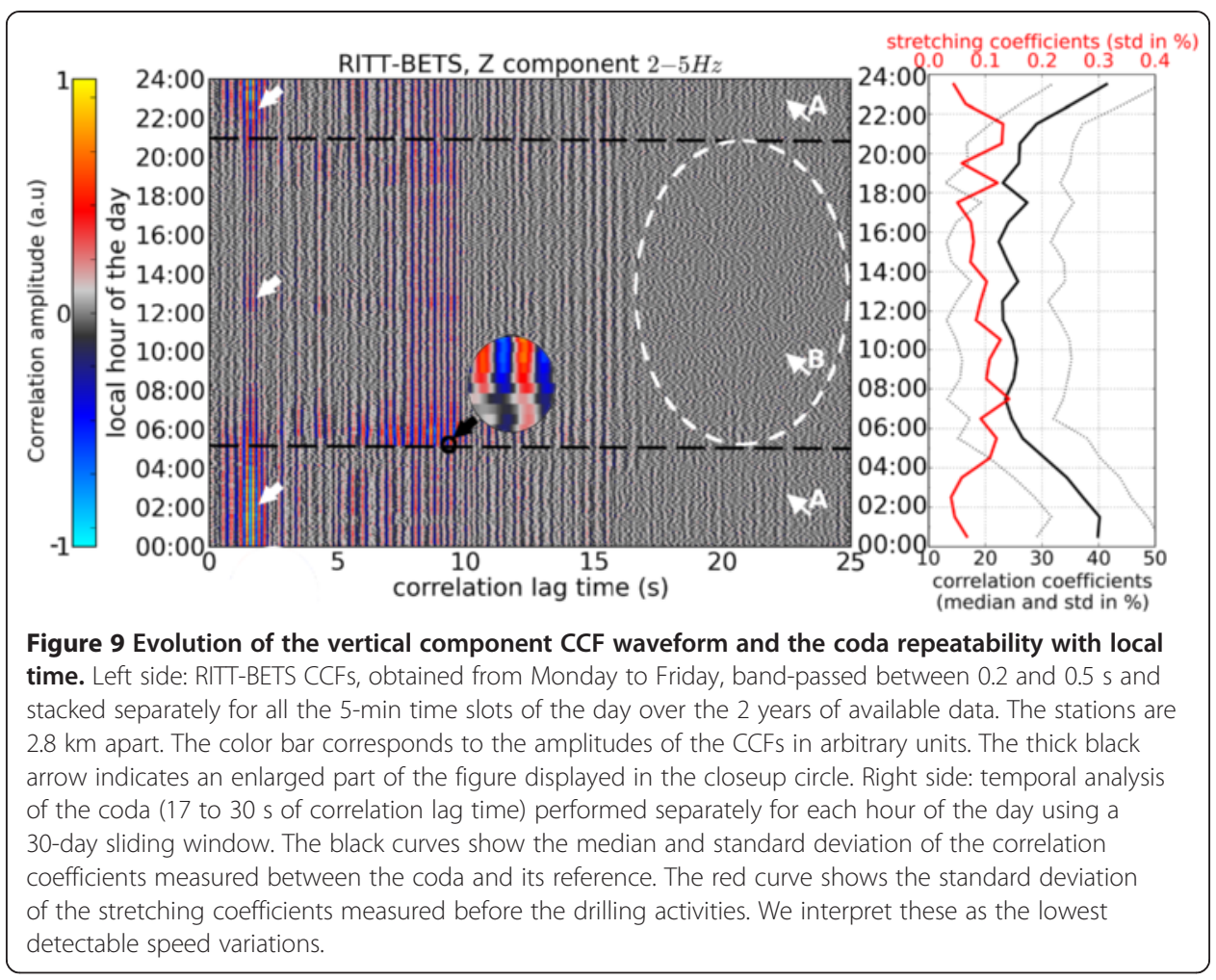

the chosen period range ( 0.2 to $0.5 \mathrm{~s})$, the distance separating the stations $(2.8 \mathrm{~km})$, and an estimated group speed ranging from about 0.18 to $0.3 \mathrm{~km} / \mathrm{s}$, we expect fundamental-mode Rayleigh waves to occur between 9 and $16 \mathrm{~s}$ of correlation lag time. Earlier arrivals observed throughout the day may be caused by clustered noise sources that are not aligned with the axis formed by the pair of stations. Such sources generate wave fields that reach the two stations with delay times shorter than the Rayleigh wave propagation time from one first station to the other. Interpreting these phases as part of Green's function would lead to an over-estimation of the group speed between the stations.

The late part of the correlation function (coda, e.g., after $16 \mathrm{~s}$ in the RITT-BETS case; Figure 9, left side) results from diffuse wave fields recorded coherently at both stations (seismic waves refracted on scatterers while traveling from one station to the other). Sens-Schönfelder and Wegler (2006) proposed to study the variability of the CCF coda over time to highlight velocity changes within the medium. This technique first establishes a reference coda by averaging the CCFs on a time span over which the medium is assumed to be invariant. Then, the CCFs computed on a sliding window are compared to this reference in order to identify infinitesimal variations (waveform stretching) of the coda. Obviously, the method requires the coda to be extremely repeatable so that any modification in its waveform can be attributed to changes into the medium.

We observe that the coda part of the CCF seems more stable during the night than during the day (Figure 9, left side). The early part of the coda (i.e., between 17 and $25 \mathrm{~s}$, arrows labeled 'A') displays similar waveforms from 10 p.m. to 4 a.m., while no coherent phases can be seen in this part of the coda from about 8 a.m. to 9 p.m. (white 
dashed circle and arrow labeled ' $\mathrm{B}$ '). We infer that the positions of diurnal sources change more than those of nocturnal sources within our time resolution of $5 \mathrm{~min}$. The daytime coda of the CCFs results from illuminating the scatterers around the station pair in a randomly time-varying manner, making it less repeatable.

In order to determine which part of the day is most suited for temporal analysis of the medium, we quantify the repeatability of the coda over time using the techniques of Sens-Schönfelder and Wegler (2006) and Brenguier et al. (2008). We conduct this analysis separately for each hour of the day. We first calculate 24 reference CCFs by averaging the CCFs separately as a function of local hour over the whole acquisition period. Then, for each local hour, we estimate how the coda computed over a 30-day sliding window resembles its reference CCF. Finally, for each local hour, we obtain a set of stretching coefficients (SCs) and their corresponding correlation coefficients (CCs). Medians and standard deviations of the CCs are used as indicators of coda repeatability (for instance, a value of $100 \% \pm 0 \%$ would correspond to a coda that always matches its reference whatever the position of the 30-day window). Results obtained in the RITT-BETS case, using the 17- to 30-s coda filtered between 0.2 and $0.5 \mathrm{~s}$ ( 2 to 5 $\mathrm{Hz}$ ), are presented in Figure 9 (right side, black curves). The coda is confirmed to be more stable at nighttime (up to $40 \% \pm 10 \%$ correlation between the coda and its reference). The standard deviation of the SCs obtained before the first drilling (190 days) is also displayed as a function of local hour (Figure 9, right side, red curve). This curve is used as an indicator of the smallest detectable relative speed variation $(\Delta v / v)$ that could be observed using our data set with a temporal resolution of 30 days. The detectable speed variation is about $0.1 \%$ during the day and $0.05 \%$ at night.

\section{Conclusions}

In this work, we benefited from the high station density available close to the two geothermal sites of Soultz-sous-Forêts and Rittershoffen and the long duration of available data (up to 4 years). We propose a detailed analysis of the seismic noise recorded in the area. The period range of interest is constrained by the dimension of the targeted structures. Based on the estimated seismic velocity model of the area, investigating the first $5 \mathrm{~km}$ of the crust requires working at periods between 0.2 and $5 \mathrm{~s}$, which include seismic noise that has various origins and properties. At periods above $1 \mathrm{~s}$, the secondary micro-seismic peak dominates the signal. This peak is characterized by a strong directivity (approximately $300^{\circ}$ back azimuth) in good agreement with its origin (northern part of the Atlantic Ocean). At periods below $1 \mathrm{~s}$, the noise has clear daily and weekly periodicities, which indicate its anthropogenic origin. The spatial analysis of this noise reveals that the sources are numerous but clustered around a few zones that roughly correspond to the densely populated villages of the area.

We compute CCFs for all station pairs of the network and analyze two of their properties. Firstly, we examine how the CCFs resemble Green's functions in terms of signal-to-noise ratio (SNR), dispersive behavior, and phase. This property of the CCFs is required for modeling the geographical distributions of seismic velocities (tomography), which will lead to better knowledge of the geological structures and characterization of the geothermal reservoir. At periods above $1 \mathrm{~s}$, the SNR is low and the spatial distribution of the (mainly anthropogenic) noise sources limits our ability to reconstruct Green's function, making dispersion measurements difficult. At periods 
below $1 \mathrm{~s}$, the SNR is higher. However, the high directivity of the noise at these periods affects the phase of the CCFs in a way that cannot be neglected. We expect CCFs to provide reliable information about distributions of seismic velocities inside the reservoir only if accurate knowledge of the noise directivity and rigorous estimates of errors induced on the phase are taken into account. This issue will be addressed in a forthcoming study.

Secondly, we analyze the stability of the correlation functions in time. This property is commonly used to follow the temporal variations of seismic velocities at depth and does not require a perfect match between the CCF waveform and the true Green's function. This technique is expected to provide information about changes that could occur inside the reservoir due to geothermal activities (relative displacement of scatterers induced by pressure variations, thermal fluctuations, variations of the fluid content, etc.). We show that high-frequency noise $(0.2$ to $0.5 \mathrm{~s})$ due to anthropogenic activity is more stable/repeatable at night. The nocturnal noise sources, although nonuniformly distributed, seem to be more stable in space and time, making nocturnal CCFs more suited for temporal analysis. With a time resolution of 30 days, we estimate the smallest detectable relative phase speed variation to be about $0.05 \%$ to $0.1 \%$. Future work will focus on the temporal variations of the medium induced by the operations conducted at the geothermal sites (drilling, injection/production tests, etc.).

\section{Additional files}

Additional file 1: Supplementary materials. This file contains a discussion of spectrograms and pre-processing of the noise.

Additional file 2: Figure S1. Long-term spectrograms measured on vertical component of stations LAMP (top, settled away from any village), RITT (middle, located about $1 \mathrm{~km}$ away from the nearest village), and BETS (bottom, located very close to a village). The spectra are computed per $24 \mathrm{~h}$. The color corresponds to the amplitude of the Fourier spectrum of the ground acceleration expressed in decibels $\left(20 \log \left(\mathrm{m} \cdot \mathrm{s}^{-2} \cdot \mathrm{Hz}^{-1}\right)\right)$; the color scale is defined in the same manner for the three stations in order to facilitate comparison. The white dashed lines delimitate the noise dominated by anthropogenic noise (below the line) from the one dominated by the secondary micro-seismic peak (above the line).

Additional file 3: Figure S2. Evolution of the RITT-BETS causal CCF, computed on vertical components over 2 years of data in the 0.2 - to 5 -s period band. The CCF is computed on a 1-week-long sliding window with a 1-day sliding step. Five processing schemes are compared to understand how the order in which temporal (ONEB) and spectral $(\mathrm{WH})$ normalizations are applied affects the CCF stability. The blue circles show a high-frequency signal occurring close to the 0 correlation lag time regardless of the processing method. This signal corresponds to the two drilling stages performed on the Rittershoffen platform. The red circles indicate a sudden frequency change observed on both ONEB and ONEBWH methods.

\section{Competing interests}

The authors declare that they have no competing interests.

\section{Authors' contributions}

ML participated in data acquisition, performed the data processing and analysis and drafted the manuscript. JV designed and coordinated the study, and participated in data acquisition. JS and AM participated in the design and coordination of the study. JS also contributed to the data acquisition. All authors have read and approved the final manuscript.

\section{Acknowledgements}

This work has been published under the framework of the LABEX ANR-11-LABX-0050_G-EAU-THERMIE-PROFONDE and benefits from a funding from the state managed by the French National Research Agency as part of the Investments for the future program. ML is funded by Groupe Electricite de Strasbourg. We thank GEIE EMC, ECOGI and EOST for providing the data of the permanent network. The array equipment (ARIT and AKUL) was supplied by the SisMob component of the RESIF National Research Infrastructure. We thank the Geophysical Instrument Pool Potsdam (GFZ) for providing temporary stations, as well as E. Gaucher (KIT), V. Maurer (ES-G), H. Wodling, H. Jund, and M. Grunberg (EOST) who deployed the stations and collected the data. We are grateful to the three anonymous reviewers for their constructive criticisms that greatly helped improve the content of this manuscript. 
Received: 30 May 2014 Accepted: 2 December 2014

Published online: 01 February 2015

\section{References}

Bensen GD, Ritzwoller MH, Barmin MP, Levshin AL, Lin F, Moschetti MP, Shapiro NM, Yang Y (2007) Processing seismic ambient noise data to obtain reliable broad-band surface wave dispersion measurements. Geophys J Int 169(3):1239-1260, doi:10.1111/j.1365-246X.2007.03374.x

Bonnefoy-Claudet S, Cotton F, Pierre-Yves B (2006) The nature of noise wavefield and its applications for site effects studies: a literature review. Earth Sci Rev 79(3-4):205-227, doi:10.1016/j.earscirev.2006.07.004

Brenguier F, Shapiro NM, Campillo M, Ferrazzini V, Duputel Z, Coutant O, Nercessian A (2008) Towards forecasting volcanic eruptions using seismic noise. Nat Geosci 1(2):126-130

Brenguier F, Clarke D, Aoki Y, Shapiro NM, Campillo M, Ferrazzini V (2011) Monitoring volcanoes using seismic noise correlations. Comptes Rendus Geosci 343(8-9):633-638, doi:10.1016/j.crte.2010.12.010

Bussat S, Kugler S (2011) Offshore ambient-noise surface-wave tomography above $0.1 \mathrm{~Hz}$ and its applications. Leading Edge 30(5):514-524, doi:10.1190/1.3589107

Calò M, Dorbath CC (2013) Different behaviours of the seismic velocity field at Soultz-sous-Forêts revealed by 4-D seismic tomography: case study of GPK3 and GPK2 injection tests. Geophys J Int 194(2):1119-1137

Calò M, Dorbath C, Cornet FH, Cuenot N (2011) Large-scale aseismic motion identified through 4-DP-wave tomography. Geophys J Int 186(3):1295-1314

Calò M, Kinnaert X, Dorbath C (2013) Procedure to construct three-dimensional models of geothermal areas using seismic noise cross-correlations: application to the Soultz-sous-Forêts enhanced geothermal site. Geophys J Int 194(3):1893-1899, doi:10.1093/gji/ggt205

Campillo M, Sato H, Shapiro NM, van der Hilst RD (2011) Nouveaux Développements de L'imagerie et Du Suivi Temporel à Partir Du Bruit Sismique. Comptes Rendus Geosci 343(8-9):487-495, doi:10.1016/j.crte.2011.07.007

Derode A, Larose E, Campillo M, Fink M (2003a) How to estimate the Green's function of a heterogeneous medium between two passive sensors? Application to acoustic waves. Appl Phys Lett 83(15):3054-3056, doi:10.1063/1.1617373

Derode A, Larose E, Tanter M, de Rosny J, Tourin A, Campillo M, Fink M (2003b) Recovering the Green's function from field-field correlations in an open scattering medium (L). J Acoust Soc Am 113(6):2973-2976, doi:10.1121/1.1570436

Dziewonski A, Bloch S, Landisman M (1969) A technique for the analysis of transient seismic signals. Bull Seismol Soc Am 59(1):427-444

Froment B, Campillo M, Roux P, Gouédard P, Verdel A, Weaver RL (2010) Estimation of the effect of nonisotropically distributed energy on the apparent arrival time in correlations. Geophysics 75(5):SA85-SA93, doi:10.1190/1.3483102

Gaucher E, Maurer V, Wodling H, Grunberg M (2013) Towards a dense passive seismic network over Rittershoffen geothermal field. 2nd European Geothermal Workshop. KIT, EOST, Strasbourg, France

Gouédard P, Roux P, Campillo M, Verdel A (2008) Convergence of the two-point correlation function toward the Green's function in the context of a seismic-prospecting data set. Geophysics 73(6):V47-V53, doi:10.1190/1.2985822

Groos JC, Ritter JRR (2009) Time domain classification and quantification of seismic noise in an urban environment. Geophys J Int 179(2):1213-1231

Gutenberg B (1936) On microseisms. Bull Seismol Soc Am 26(2):111-117

Hadziioannou C, Larose E, Coutant O, Roux P, Campillo M (2009) Stability of monitoring weak changes in multiply scattering media with ambient noise correlation: laboratory experiments. J Acoust Soc Am 125(6):3688-3695

Kedar S, Longuet-Higgins M, Webb F, Graham N, Clayton R, Jones C (2008) The origin of deep ocean microseisms in the North Atlantic Ocean. Proc Royal Soc A Math Phys Eng Sci 464(2091):777-793, doi:10.1098/rspa.2007.0277

Larose E (2005) Diffusion multiple des ondes sismiques et expériences analogiques en ultrasons. Université Joseph-Fourier - Grenoble I, France

Larose E, Roux P, Campillo M (2007) "Reconstruction of Rayleigh-Lamb Dispersion Spectrum Based on Noise Obtained from an Air-Jet Forcing". The Journal of the Acoustical Society of America 122(6):3437-44. doi:10.1121/1.2799913

Lin F-C, Tsai VC (2013) Seismic interferometry with antipodal station pairs. Geophys Res Letts 40(17):4069-4613. doi:10.1002/grl.50907

Lin F-C, Tsai VC, Schmandt B, Duputel Z, Zhan Z (2013) Extracting seismic core phases with array interferometry. Geophys Res Lett 40(6):1049-1053, doi:10.1002/grl.50237

Lobkis Ol, Weaver RL (2001) On the emergence of the Green's function in the correlations of a diffuse field. J Acoust Soc Am 110(6):3011-3017, doi:10.1121/1.1417528

Longuet-Higgins MS (1950) A theory of the origin of microseisms. Phil Trans R Soc A 243(857):1-35, doi:10.1098/ rsta.1950.0012

McNamara DE, Buland RP (2004) Ambient noise levels in the continental United States. Bull Seismol Soc Am 94(4):1517-1527

Mordret A, Landés M, Shapiro NM, Singh SC, Roux P, Barkved OI (2013) Near-surface study at the Valhall oil field from ambient noise surface wave tomography. Geophys J Int 193(3):1627-1643, doi:10.1093/gji/ggt061

Obermann A, Planès T, Larose E, Campillo M (2013) Imaging preeruptive and coeruptive structural and mechanical changes of a volcano with ambient seismic noise. J Geophys Res Solid Earth 118(12):6285-6294, doi:10.1002/2013JB010399

Pedersen HA, Krüger F (2007) Influence of the seismic noise characteristics on noise correlations in the Baltic shield. Geophys J Int 168(1):197-210

Poli P, Campillo M, Pedersen H (2012) Body-wave imaging of Earth's mantle discontinuities from ambient seismic noise. Science 338(6110):1063-1065, doi:10.1126/science.1228194

Rost S, Thomas eC (2002) Array seismology: methods and applications. Rev Geophys 40(3):1008, doi:10.1029/2000RG000100

Roux P, Kuperman WA, and the NPAL Group (2004) Extracting coherent wave fronts from acoustic ambient noise in the ocean. J Acoust Soc Am 116(4):1995-2003, doi:10.1121/1.1797754

Roux P, Sabra KG, Kuperman WA, Roux A (2005) Ambient noise cross correlation in free space: theoretical approach. J Acoust Soc Am 117(1):79-84, doi:10.1121/1.1830673

Sabra KG, Gerstoft P, Roux P, Kuperman WA, Fehler MC (2005a) Extracting time-domain Green's function estimates from ambient seismic noise. Geophys Res Lett 32(3):L03310 
Sabra KG, Gerstoft P, Roux P, Kuperman WA, Fehler MC (2005b) Surface wave tomography from microseisms in southern California. Geophys Res Lett 32(14):L14311

Sabra KG, Roux P, Kuperman WA (2005c) Arrival-time structure of the time-averaged ambient noise cross-correlation function in an oceanic waveguide. J Acoust Soc Am 117(1):164-174

Sens-Schönfelder C, Wegler U (2006) Passive image interferometry and seasonal variations of seismic velocities at Merapi Volcano, Indonesia. Geophys Res Lett 33(21):L21302, doi:10.1029/2006GL027797

Sergeant A, Stutzmann E, Maggi A, Schimmel M, Ardhuin F, Obrebski, M (2013) "Frequency-Dependent Noise Sources in the North Atlantic Ocean." Geochem Geophys Geosyst 14(12):5341-5353, doi:10.1002/2013GC004905

Shapiro NM, Campillo M (2004) Emergence of broadband Rayleigh waves from correlations of the ambient seismic noise. Geophys Res Lett 31(7):L07614

Shapiro NM, Campillo M, Stehly L, Ritzwoller MH (2005) High-resolution surface-wave tomography from ambient seismic noise. Science 307(5715):1615-1618, doi:10.1126/science.1108339

Stehly L, Campillo M, Shapiro NM (2006) A study of the seismic noise from its long-range correlation properties. $J$ Geophys Res 111(B10):B10306

Weaver R, Froment B, Campillo M (2009) On the correlation of non-isotropically distributed ballistic scalar diffuse waves. J Acoust Soc Am 126(4):1817-1826, doi:10.1121/1.3203359

Weaver RL, Hadziioannou C, Larose E, Campillo M (2011) On the precision of noise correlation interferometry. Geophys J Int 185(3):1384-1392, doi:10.1111/j.1365-246X.2011.05015.x

Withers MM, Aster RC, Young CJ, Chael EP (1996) High-frequency analysis of seismic background noise as a function of wind speed and shallow depth. Bull Seismol Soc Am 86(5):1507-1515

Submit your manuscript to a SpringerOpen ${ }^{\mathcal{O}}$ journal and benefit from:

- Convenient online submission

Rigorous peer review

- Immediate publication on acceptance

- Open access: articles freely available online

- High visibility within the field

- Retaining the copyright to your article

Submit your next manuscript at $\boldsymbol{\nabla}$ springeropen.com 